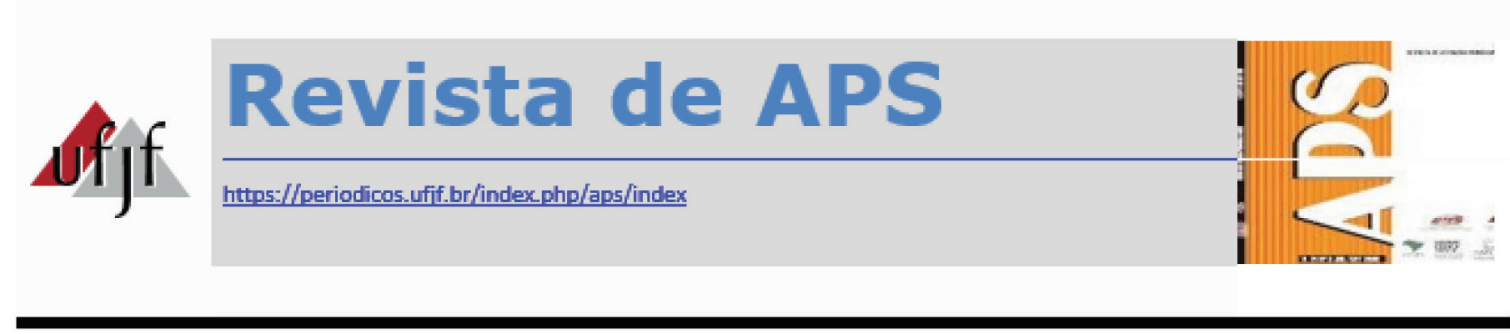

\title{
Terapia comunitária integrativa na abordagem de transtornos mentais comuns na atenção primária à saúde: uma revisão sistemática
}

\section{Community integrative therapy for common mental disorders in Primary Health Care: a systematic review}

Alessandro da Silva Scholze ${ }^{1}$

\section{RESUMO}

A Atenção Primária à Saúde (APS) constitui a porta preferencial de acesso ao Sistema de Saúde, devendo atender com resolutividade a maior parcela dos problemas de saúde, incluindo os transtornos mentais comuns (TMC). A Terapia Comunitária Integrativa (TCI) é uma ferramenta desenvolvida como um recurso para pessoas com sofrimentos de qualquer natureza, especialmente psicossociais, sendo reconhecida como tal e promovida no Sistema Único de Saúde para aplicação em cenários diversos, desde a APS até serviços de especialidades focais. O objetivo desta revisão sistemática da literatura foi buscar evidências que permitam avaliar a efetividade da TCl como forma de abordagem para pessoas com TMC na APS. A busca realizada para estudos publicados nos últimos 10 anos com o termo "Terapia Comunitária" na Bireme e "Community Therapy" no PubMed identificou, juntamente com outras fontes, 169 registros não duplicados. Após análise inicial dos títulos e resumos desses registros de acordo com os critérios de inclusão e exclusão, foram analisados os textos completos de 14 estudos, nenhum dos quais avaliava a efetividade da TCl de forma longitudinal, comparando os resultados desta com quaisquer outras modalidades de intervenção. Assim, não há evidências na literatura atual que apontem para a efetividade da TCI na abordagem dos TMC na APS. Sugere-se a necessidade do desenvolvimento de pesquisas com metodologia adequada para responder a essa questão e prover maior suporte teórico para a base empírica da TCl.

PALAVRAS-CHAVE: Assistência à saúde. Redes comunitárias. Transtornos mentais. Terapêutica.

${ }^{1}$ Médico de Família e Comunidade. Mestre em Saúde e Gestão do Trabalho. Docente do Curso de Medicina da UnivaliItajaí/SC. E-mail: alescholze@gmail.com 


\begin{abstract}
Primary Care (PC) services should be the first contact for resolutive care in the health system, including for people suffering of Common Mental Disorders (CMD). Community Integrative Therapy (CIT) is a tool developed as a resource for people with any kind of suffering, particularly psychosocial suffering, and provided by the Brazilian Health System (SUS) in different settings, as PC and secondary care services. This systematic review aimed to search evidences demonstrating CTI's effectivity in providing care for people with CMD in PC. The search was conducted for studies published in the last 10 years using the terms "Terapia Comunitária" ("Community Therapy") in Bireme and "Community Therapy" in PubMed. Considering these databases and other sources, there were 169 non-duplicated registries. After the previous analyses of titles and abstracts for inclusion and exclusion criteria, we found 14 studies that were taken to full text analysis. But neither of them was conducted to verify CTI's effectivity with a longitudinal design and comparing it with other forms of intervention. So, there are no current evidences to confirm or deny CTI's effectivity in providing care for people with CMD in PC. We suggest the need of new research projects to study that this question with adequate methodology in order to give a stronger theoretical support for CTI's empirical fundaments.
\end{abstract}

KEYWORDS: Delivery of Heath Care. Community Networks. Mental Disorders. Therapeutics.

\title{
INTRODUÇÃO
}

Os transtornos mentais atingem $29,2 \%$ da população em algum momento ao longo da vida. Uma a cada cinco pessoas na população geral apresenta algum transtorno mental comum (TMC), incluindo transtornos de humor depressivo ou bipolar, transtornos de ansiedade, transtornos por uso de álcool ou outras substâncias (usuários problema ou dependentes) ${ }^{1}$. Metade das pessoas afetadas têm sintomas significativos a ponto de serem levados à atenção de um profissional de saúde. Na maioria dos casos são encontrados sintomas mistos de depressão e ansiedade ${ }^{2}$.

Muitas pessoas buscam os serviços de saúde por sofrimento mental, geralmente com queixas de tristeza e/ou ansiedade, as quais, por vezes, mesmo implícitas, também são frequentemente identificadas pelos profissionais da Atenção Primária em Saúde (APS). Tais sintomas, reconhecidos como depressivos, ansiosos e de somatização (ou queixas físicas sem explicação médica), constituem as manifestações mais comuns do sofrimento mental na APS e podem ser consideradas como parte de uma única síndrome clínica com três grupos ou dimensões de sintomas que se combinam, denominadas transtornos mentais comuns (TMC) ${ }^{3}$.

Uma revisão sistemática de 174 pesquisas verificou que 17,6\% dos adultos haviam sofrido algum TMC no último ano, sendo a incidência ao longo da vida de 29,2\%. Entre 26 países de maior renda e outros 37 de renda média ou baixa demonstrou-se uma heterogeneidade substancial na prevalência de transtornos mentais comuns, porém em ambos os grupos as mulheres estavam mais propensas a desenvolverem transtornos 
de humor ou ansiedade, enquanto os homens experimentavam mais frequentemente problemas com o uso de álcool ou outras substâncias ${ }^{1}$.

As causas dos TMC são multifatoriais, envolvendo fatores psicológicos, sociais e biológicos. Ainda que a vulnerabilidade individual seja variável, eventos negativos na vida, incluindo os problemas de saúde em geral, parecem ter um papel no desenvolvimento desses transtornos. Assim, uma série de fatores socioeconômicos afetam a prevalência de TMC, como a depressão ser mais comum entre desempregados, com baixa renda, sem educação formal e que passaram por três ou mais mudanças de moradia nos últimos dois anos. Além disso, fatores como sexo feminino, apresentar problemas de aprendizagem, ser separado/divorciado também se correlacionam com maior probabilidade de apresentar $\mathrm{TMC}^{2}$.

Um estudo investigando as percepções sobre estresse, distresse e experiência da doença em quatro clínicas de Atenção Primária diferentes na Inglaterra, que incluiu 268 participantes sendo 117 com algum transtorno mental, verificou que as pessoas com sofrimento mais intenso eram as mais propensas a buscar o médico na AP, bem como são as que mais utilizavam outros recursos de cuidado como a família ou o sistema popular de práticas tradicionais de cuidado. Em relação ao cuidado médico no sistema formal, este era visto como pouco resolutivo quando o médico parecia pouco responsivo às preocupações da pessoa, estava muito focado no tratamento medicamentoso ou porque a pessoa via seu problema como sem solução. Por outro lado, as percepções positivas do cuidado médico eram trazidas por pessoas que tinham baixas expectativas em relação ao serviço de saúde ou haviam obtido melhora com o tratamento médico 4 .

Em revisão integrativa sobre ações em saúde mental desenvolvidas pela enfermagem e voltadas às famílias, sugeriu-se que os estudos com enfoque na Atenção Básica apontavam para a necessidade de maior preparo dos profissionais, considerandose seu papel central a partir da reforma psiquiátrica, uma vez que se identifica ainda uma orientação do trabalho das equipes na APS com base na psiquiatria tradicional e medicalização do sofrimento psíquico. Tais ações deveriam ser repensadas, buscando-se a inclusão de tecnologias leves como dispositivos para a produção do cuidado para os usuários e suas famílias ${ }^{5}$.

A Terapia Comunitária Integrativa ( $\mathrm{TCl}$ ) é proposta como uma forma de abordagem do sofrimento psicossocial. A TCl é realizada em grupos abertos, conduzidos por um a três terapeutas com formação específica para isso, desenvolvendo um espaço de acolhimento no qual se propõe a escuta do sofrimento e o resgate das potencialidades da comunidade a fim de lidar com tais situações. Dados de 12.000 questionários realizados durante os anos de 2005 e 2006 identificavam como tema mais frequente nas rodas de $\mathrm{TCl}$ a categoria do estresse $(26,7 \%)$, que inclui a ansiedade e sintomas somáticos, sendo a depressão também comumente identificada como categoria do tema, em 9,3\% das rodas ${ }^{6}$. 
A Portaria № 849, de 27 de março de 2017 incluiu a Terapia Comunitária Integrativa ( $\mathrm{TCl}$ ) entre as práticas estabelecidas nas diretrizes da Política Nacional de Práticas Integrativas e Complementares no Sistema Único de Saúde ${ }^{7}$. Antes mesmo dessa inclusão, a $\mathrm{TCl}$ já era reconhecida no Caderno de Atenção Básica de Saúde Mental como uma ferramenta a ser utilizada pelos profissionais da Atenção Básica. No território, qualquer pessoa que tenha interesse poderia participar da $\mathrm{TCl}$, como um espaço que possibilita a troca de experiências e de sabedorias de vida, tornando todos os participantes corresponsáveis pelo processo terapêutico que produz efeitos tanto grupais quanto singulares, permitindo o reconhecimento de uma rede de apoio na comunidade ${ }^{3}$.

Barreto definiu a $\mathrm{TCl}$ como "[...] um espaço de promoção de encontros interpessoais e intercomunitários, objetivando a valorização das histórias de vida dos participantes, o resgate da identidade, a restauração da autoestima e da confiança em si, a ampliação da percepção dos problemas e possibilidades de resolução a partir das competências locais. Tem como base de sustentação o estímulo para a construção de vínculos solidários e promoção de vida"6:33.

A TCl foi desenvolvida com base em cinco grandes eixos teóricos: Pensamento Sistêmico, Teoria da Comunicação, Antropologia Cultural, pedagogia de Paulo Freire e Resiliência. A partir desse arcabouço teórico, a TCl se propõe a superar o modelo centrado na patologia, direcionando-se para a promoção da saúde, através da construção de redes de solidariedade e inclusão social. Diferente de um processo psicoterapêutico, a $\mathrm{TCl}$ se constitui em um ato terapêutico de grupo dirigida por terapeutas que se colocam como facilitadores, devidamente treinados. Suas intervenções enfocam as redes de relações humanas, como a família e os vizinhos, empregando a coletividade para apoiar os mais vulneráveis da comunidade que vivem uma situação de crise ${ }^{6}$.

Lazarte refletiu sobre como a $\mathrm{TCl}$ promove a mobilização social, dissolvendo as barreiras que separam as pessoas em subgrupos, seja pela cultura ou pela classe social, eliminando "pré-conceitos", no que seria uma de suas interfaces mais evidentes e positivas com a sociologia, no sentido da eliminação sistemática de todas as pré-noções, proposta por Durkheim como uma das regras do método sociológico. No sentido da participação social, a $\mathrm{TCl}$ restitui a identidade da pessoa, permitindo um reencontro consigo mesmo, a partir do que são reforçados os laços sociais, agregando pessoas e comunidades. $\mathrm{O}$ aspecto integrativo da $\mathrm{TCl}$ permite que as pessoas percebam a unidade das suas vidas e, à medida que a história pessoal de cada um e de cada uma vem à tona, ela também se emparenta com as histórias de vida dos outros presentes, favorecendo a inclusão social. Por fim, em uma convergência com o pensamento de Karl Marx, o processo de autoconhecimento que se verifica nas rodas da Terapia Comunitária conduz a uma desalienação, ou seja, o retorno da pessoa ao seu ser original, abandonando imagens falsas ou equivocadas a respeito de si mesma ${ }^{8}$. 
A $\mathrm{TCl}$ como objeto de pesquisa científica é bem mais recente que sua prática, identificando-se os primeiros artigos publicados em periódicos indexados no ano de 2006. Uma revisão integrativa da produção científica brasileira sobre $\mathrm{TCl}$ identificou 48 trabalhos, os quais privilegiavam os aspectos de promoção da saúde, utilizando metodologias qualitativas em $84 \%$ dos casos e tendo como cenários predominantemente as Unidades Básicas de Saúde (35\%) e os Centros de Atenção Psicossocial (37\%) . Ferreira Filha, Lazarte e Barreto sugeriram que a predominância de pesquisas qualitativas, em especial com o método de pesquisa ação, reflete um aspecto da própria $\mathrm{TCl}$ ao buscar resgatar as experiências pessoais em uma outra fonte de conhecimento, mais intuitiva e cultural, sem negar o saber acadêmico quantitativo, mas rejeitando seu monopólio ${ }^{10}$.

Em uma revisão integrativa desenvolvida a partir da questão “Qual é a contribuição da terapia comunitária no processo saúde-doença, segundo as produções científicas?", foram incluídos cinco estudos, cujos sujeitos eram predominantemente mulheres e idosos, tanto na APS como em outros contextos. As metodologias empregadas foram qualitativas, demonstrando que a $\mathrm{TCl}$ proporcionava melhora da autoestima, maior segurança na tomada de decisões, reintegração social e fortalecimento dos vínculos familiares, de amizade e espirituais. Os participantes descreveram os encontros como sendo marcados pelos sentimentos de alívio e alegria, pelo apoio, pela troca de experiências a busca de respostas para a solução dos problemas ${ }^{11}$.

A prática da $\mathrm{TCl}$ na Unidade Básica de Saúde permite a criação de um espaço grupal permanente de apoio e ajuda mútua para a comunidade, proporcionando uma sensibilização quanto aos problemas coletivos, outrora desconhecidos. Cada participante pode se sentir acolhido em sua experiência de doença, socializando também formas de superação, a partir do que se pode observar uma redução na busca por consultas e medicamentos ${ }^{12}$.

Um relato de experiência sobre a $\mathrm{TCl}$ em uma UBS verificou que os sentimentos mais comumente apresentados nos relatos das rodas foram medo, preocupação, ansiedade, angústia, frustração e insatisfação. No relato dos participantes sobre a $\mathrm{TCl}$, evidenciaram-se sentimentos positivos, sugerindo o potencial dessa terapia para o enfrentamento do sofrimento psíquico na APS e como ação de prevenção a transtornos mais graves ${ }^{13}$.

Em estudo qualitativo para analisar as opiniões dos profissionais de saúde e de usuários em relação à TCI realizado em uma Unidade de Saúde da Família em Pernambuco, que contava com essa terapia há três anos, a opinião dos grupos de profissionais e usuários concordava sobre a $\mathrm{TCl}$ como sendo um espaço de fala e de escuta que facilita o relacionamento entre as pessoas. Tanto profissionais como usuários, em sua maioria, responderam ter se tornado mais sociáveis e comunicativos, ao mesmo tempo em que desenvolveram empatia e maior atenção na escuta do outro. Assim, a TCl promoveu o autocontrole, tornando as pessoas mais calmas e pacientes 
com as diferenças individuais, especialmente nas relações familiares, e melhorando a confiança nos profissionais de saúde ${ }^{14}$.

Ainda que uma tecnologia de baixo custo, a TCl utiliza recursos do Sistema Único de Saúde, o qual tem entre seus princípios a equidade. A fim de utilizar esses recursos limitados de forma adequada às necessidades e demandas em saúde da população, profissionais e gestores devem contar com tecnologias avaliadas e validadas. A TCI foi desenvolvida no final do século passado pelo médico Adalberto de Paula Barreto, sistematizada em 1987 e desde então tem sido amplamente utilizada no Brasil e em outros países, sendo também objeto de diversas pesquisas, mas ainda busca consolidarse a partir de um arcabouço de conhecimento científico.

Nesse sentido, não são identificadas na literatura Revisões Sistemáticas que demonstrem sua efetividade na abordagem de TMC na Atenção Primária à Saúde. Assim, neste estudo, realizamos uma revisão sistemática da literatura buscando identificar evidências que permitam avaliar a efetividade da $\mathrm{TCl}$ como forma de abordagem para pessoas com transtornos mentais comuns na APS.

\section{DESENVOLVIMENTO}

A pergunta de pesquisa, no formato PICO (população/intervenção/comparação/ objetivo), foi colocada da seguinte forma:

$\mathrm{P}$ - Pessoas com transtornos mentais comuns na APS

I - Terapia comunitária integrativa

C - Outras formas de tratamento farmacológico ou não farmacológico

O - Melhora sintomática/redução na demanda nos serviços de saúde/redução no uso de psicofármacos

A revisão dos estudos sobre $\mathrm{TCI}$ na abordagem dos TMC foi realizada de acordo com os critérios a seguir ${ }^{15}$.

\section{Desenho do estudo}

Estudos quantitativos ou qualitativos, prospectivos ou retrospectivos, que utilizem a TCl como uma forma de intervenção na abordagem de pessoas com transtornos mentais comuns na APS em comparação com outras formas de intervenção, seja em forma de estudos clínicos controlados randomizados ou não randomizados, casos-controles ou controles históricos. Serão excluídas séries de casos sem controles, estudos transversais e relatos de casos. 


\section{Participantes}

Pessoas com transtornos mentais comuns na APS, sem restrição de gênero ou idade, podendo ou não apresentar outras comorbidades relativas a problemas somáticos, tendo sido identificadas em quaisquer níveis de atenção do Sistema de Saúde para participação no estudo. Serão excluídos estudos direcionados a pessoas com outros transtornos mentais ou que não apresentem avaliações relativas a problemas mentais.

\section{Intervenções}

Foram incluídos estudos nos quais a $\mathrm{TCl}$ seja uma das intervenções realizadas, em comparação com quaisquer outras formas de abordagem para pessoas com transtornos mentais comuns na APS, tanto farmacológicas como não farmacológicas, mesmo em comparação com listas de espera, em cuidados habituais ou controles históricos.

\section{Resultados}

Os resultados buscados nos estudos para as comparações entre a $\mathrm{TCl}$ e outras intervenções realizadas incluíram avaliações quantitativas e/ou qualitativas que permitam identificar, seja empregando escalas padronizadas, indicadores específicos ou descrições subjetivas:

- melhora sintomática;

- redução na demanda nos serviços de saúde;

- ou redução no uso de psicofármacos.

\section{Seguimento}

O tempo de seguimento realizado em cada estudo incluído foi identificado tendo critério de inclusão seu desenvolvimento de forma longitudinal, a fim de verificar mudanças entre o período anterior e o posterior à participação na $\mathrm{TCl}$. $\mathrm{O}$ tempo de seguimento não foi, entretanto, fator de inclusão ou exclusão de estudos para a revisão.

\section{Idioma}

Foram incluídos estudos em quaisquer idiomas. 


\section{Fontes de informação}

Os estudos foram identificados a partir das bases de dados da BIREME e PubMed. Além dos estudos identificados a partir dessas bases de dados, foram incluídos estudos referenciados nos artigos assim identificados, livros, trabalhos publicados em anais de eventos e teses ou dissertações sobre o tema "Terapia Comunitária Integrativa".

\section{Estratégia de busca dos estudos}

A busca envolveu estudos realizados entre 2007 e 2017, cobrindo o período dos últimos dez anos. O termo de busca empregado foi "Terapia Comunitária" na base de dados da BIREME, e "Community Therapy" no PubMed. Esse termo pareceu mais sensível que "Terapia Comunitária Integrativa" no momento da revisão de literatura, ao mesmo tempo em que especificava melhor a $\mathrm{TCl}$, ao excluir outras formas de terapias consideradas integrativas ou integrais.

\section{Processo de seleção}

Os autores desenvolveram um processo independente de verificação dos títulos e resumos dos estudos identificados na busca inicial, a fim de confirmar os critérios de inclusão definidos. A partir disso, foram buscados os textos completos para aqueles que fossem definidos como incluídos ou sobre os quais houvesse incerteza. Os critérios de inclusão foram, então, conferidos pelos autores nos textos completos e discutidas possíveis dúvidas a respeito daqueles. Registraram-se os critérios para exclusão dos estudos avaliados na forma de texto completo.

\section{Coleta de dados}

Os dados não chegaram a ser coletados a partir do texto completo, uma vez que não houve estudos selecionados para inclusão após a análise inicial.

O processo de busca e seleção dos estudos foi representado em um diagrama utilizando o modelo PRISMA (Figura 1$)^{15}$.

Os dados utilizados foram obtidos a partir de artigos e outras fontes publicadas, de domínio público, sem haver implicações éticas envolvendo seres humanos.

Foram identificados 136 registros de estudos pela busca do termo "Terapia Comunitária" na Bireme. No PubMed, foram identificados 15 registros de estudos pela busca do termo "Community Therapy". Uma vez selecionados apenas os estudos dos últimos 10 anos, na Bireme restaram 111 estudos, além de sete estudos no PubMed. 
Neste último, três eram identificados como revisões sistemáticas, e os outros quatro, como estudos clínicos.

Outros estudos relativos à temática foram identificados em livros, teses e dissertações (literatura cinzenta), anais de eventos e periódicos não indexados. Esses achados se deram por sua citação em obras consultadas e referências utilizadas na revisão de literatura de forma não sistemática, além de material utilizado na capacitação e especialização em $\mathrm{TCl}$. Tais estudos somaram 94 registros.

Dentre os estudos do PubMed, apenas um se referia à temática da $\mathrm{TCl}$, porém este também foi identificado na revisão da Bireme, surgindo então em duplicata. Na Bireme, 98 registros diziam respeito a textos, enquanto os outros 14 davam acesso a recursos educacionais, sendo que 79 registros disponibilizavam textos completos. Foram excluídos registros duplicados, restando 74 documentos (Figura 1). 
Quadro 1 - Estudos verificados na íntegra durante a revisão sistemática

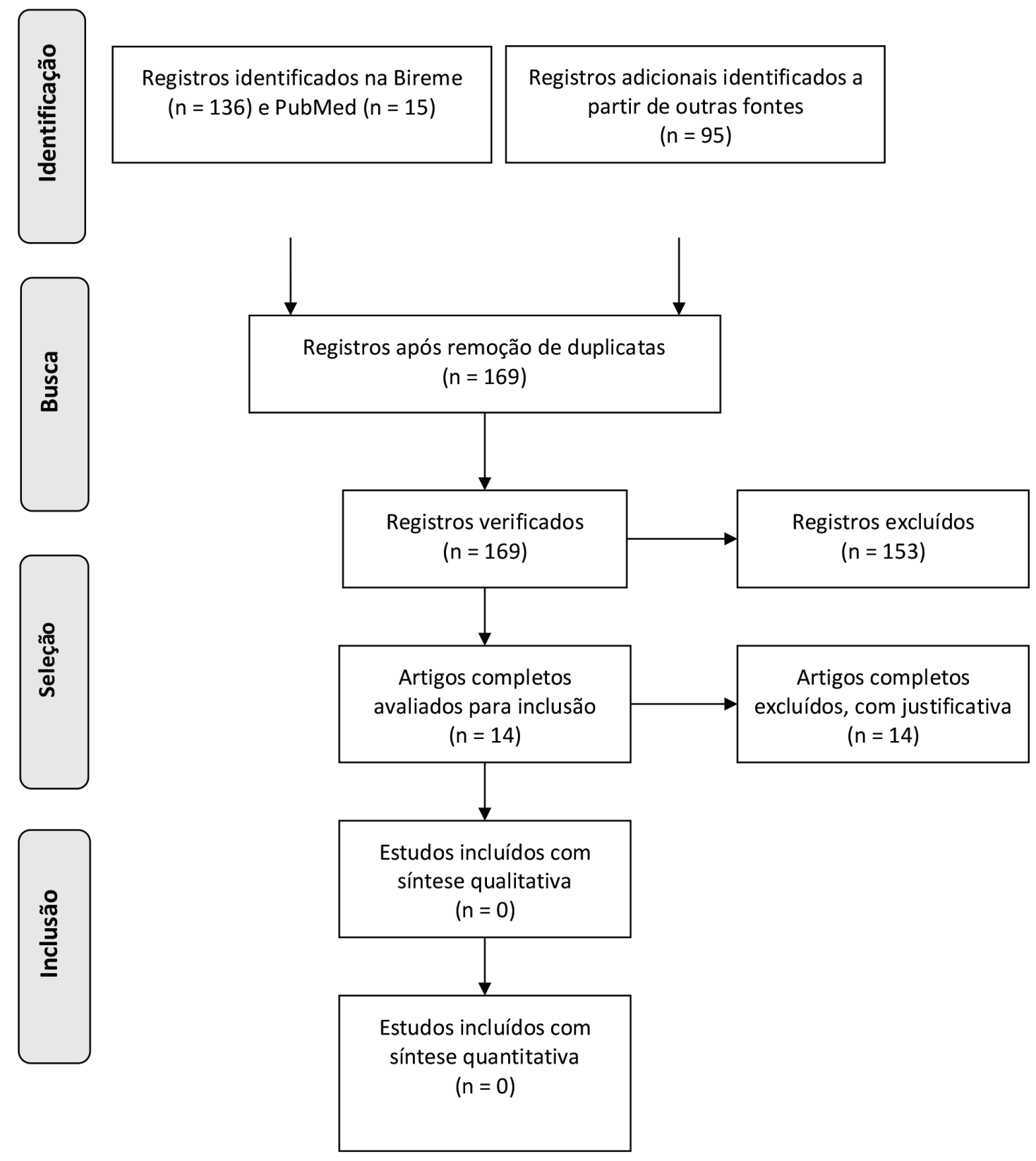

Fonte: Moher D, Liberati A, Tetzlaff J, Altman DG, The PRISMA Group (2009). Preferred Reporting Items for Systematic Reviews and Meta-Analyses: The PRISMA Statement. PLoS Med 6(7): e1000097.doi:10.1371/journal.pmed1000097 
Após a verificação dos critérios de inclusão e exclusão nos resumos dos registros de estudos com a temática da $\mathrm{TCl}$ publicados no período de abrangência da revisão, os estudos selecionados contavam com textos completos disponíveis, em inglês ou português, todos os quais foram consultados na íntegra. Destes, nenhum se enquadrava em todos os critérios da revisão sistemática proposta, sendo os motivos da exclusão detalhados no Quadro 1.

Quadro 1 - Estudos verificados na íntegra durante a revisão sistemática

\begin{tabular}{|c|c|c|c|}
\hline Estudo & Fonte & Metodologia & Motivos para exclusão \\
\hline 1. Soares, $2008^{16}$ & Outros & Qualitativa & $\begin{array}{l}\text { Transversal. Sujeitos com } \\
\text { somatização, ansiedade, } \\
\text { tristeza. Ausência de grupo } \\
\text { de comparação e avaliação } \\
\text { de resultados. }\end{array}$ \\
\hline 2. Arruda, $2011^{17}$ & Outros & Qualitativa & $\begin{array}{l}\text { Transversal. Estudo de } \\
\text { caso. Os sujeitos incluíam } \\
\text { participantes com TMC e } \\
\text { terapeutas. Ausência de } \\
\text { grupo de comparação e } \\
\text { avaliação de resultados }\end{array}$ \\
\hline $\begin{array}{l}\text { 3. Cordeiro, } \\
\text { Azevedo, Silva, } \\
\text { Ferreira Filha, } \\
\text { Silva, Moraes, } \\
2011^{18} \\
\end{array}$ & Outros & Qualitativa & $\begin{array}{l}\text { Transversal. Inclusão de } \\
\text { pessoas com HAS e DM sem } \\
\text { definição de TMC. }\end{array}$ \\
\hline $\begin{array}{l}\text { 4. Fernandes, de } \\
\text { Aquino, Santos, } \\
\text { Soares, de Lima, } \\
2011^{19}\end{array}$ & Outros & Qualitativa & $\begin{array}{l}\text { Transversal. Estudo } \\
\text { descritivo sem grupo de } \\
\text { comparação. Inclusão de } \\
\text { sujeitos sem TMC. Descreve } \\
\text { a metodologia sem avaliação } \\
\text { dos resultados. }\end{array}$ \\
\hline $\begin{array}{l}\text { 5. Moura, } \\
\text { Ferreira Filha, } \\
\text { Cordeiro, Braga, } \\
\text { Monteiro, } 2012^{20}\end{array}$ & Outros & Qualitativa & $\begin{array}{l}\text { Transversal. Incluiu subgrupo } \\
\text { de pacientes do CAPS. } \\
\text { Ausência de grupo de } \\
\text { comparação e avaliação de } \\
\text { resultados. }\end{array}$ \\
\hline $\begin{array}{l}\text { 6. NASCIMENTO, } \\
\text { et al. } 2012^{12}\end{array}$ & Outros & Qualitativa & $\begin{array}{l}\text { Relato de experiência } \\
\text { sobre a implantação da } \\
\text { TCI. Sujeitos com queixas } \\
\text { de adoecimento difuso. } \\
\text { Ausência de grupo de } \\
\text { comparação e avaliação de } \\
\text { resultados. }\end{array}$ \\
\hline
\end{tabular}


(Continuação)

\begin{tabular}{|c|c|c|c|}
\hline Estudo & Fonte & Metodologia & Motivos para exclusão \\
\hline $\begin{array}{l}\text { 7. Ferreira Filha, Sá, } \\
\text { Rocha, Silva, Souto, } \\
\text { Dias, } 2012^{21}\end{array}$ & Bireme & Qualitativa & $\begin{array}{l}\text { Transversal. Inclusão de } \\
\text { sujeitos devido a alcoolismo } \\
\text { na família, sem definição de } \\
\text { TMC. Ausência de grupo de } \\
\text { comparação e avaliação de } \\
\text { resultados. }\end{array}$ \\
\hline $\begin{array}{l}\text { 8. Rocha, Sá, Braga, } \\
\text { Ferreira Filha, Dias, } \\
2013^{22}\end{array}$ & Bireme & Qualitativa & $\begin{array}{l}\text { Transversal. Estudo } \\
\text { documental. Temas diversos } \\
\text { incluindo sofrimento } \\
\text { psíquico. Ausência de grupo } \\
\text { de comparação e avaliação } \\
\text { de resultados. }\end{array}$ \\
\hline 9. Nascimento, $2015^{23}$ & Outros & Qualitativa & $\begin{array}{l}\text { Longitudinal. Sujeitos } \\
\text { com sintomas psíquicos. } \\
\text { Avaliação dos resultados } \\
\text { com análise de conteúdo. } \\
\text { Ausência de grupo de } \\
\text { comparação. }\end{array}$ \\
\hline 10. Silva, $2016^{24}$ & Outros & Qualitativa & $\begin{array}{l}\text { Transversal. População } \\
\text { com baixa autoestima de } \\
\text { CAPS em modalidade não } \\
\text { intensiva com indicação de } \\
\text { alta. Ausência de grupo de } \\
\text { comparação e avaliação de } \\
\text { resultados }\end{array}$ \\
\hline $\begin{array}{l}\text { 11. Feitosa, Garcia } \\
\text { Filho, } 2016^{25}\end{array}$ & Outros & Qualitativa & $\begin{array}{l}\text { Longitudinal. Relato de } \\
\text { experiência com crianças } \\
\text { atendidas no CAPS. Ausência } \\
\text { de grupo de comparação e } \\
\text { avaliação de resultados. }\end{array}$ \\
\hline $\begin{array}{l}\text { 12. Lima Silva, } \\
\text { Medeiros, Guerra, et } \\
\text { al., } 2017^{26}\end{array}$ & Outros & Quantitativa & $\begin{array}{l}\text { Transversal. } \\
\text { Sujeitos idosos de CAPS } \\
\text { e CEASI com um grupo } \\
\text { composto por idosos com } \\
\text { depressão e outro de } \\
\text { controle. }\end{array}$ \\
\hline 13. Lutterbach, $2017^{27}$ & Outros & Qualitativa & $\begin{array}{l}\text { Transversal. Os sujeitos } \\
\text { incluídos eram profissionais } \\
\text { ou estagiários do CAPS. } \\
\text { A roda de TCI teve a } \\
\text { metodologia modificada } \\
\text { para coleta de dados. }\end{array}$ \\
\hline
\end{tabular}


(Conclusão)

\begin{tabular}{|l|l|l|l|}
\hline Estudo & Fonte & Metodologia & Motivos para exclusão \\
\hline 14. Silva, et al., 201728. & Outros & Qualitativa & $\begin{array}{l}\text { Transversal. População não } \\
\text { definida por TMC, incluindo } \\
\text { comunidade em geral e } \\
\text { familiares de pacientes do } \\
\text { CAPS. Ausência de grupo de } \\
\text { comparação e avaliação de } \\
\text { resultados. }\end{array}$ \\
\hline
\end{tabular}

Fonte: elaborado pelos autores

\section{CONCLUSÃO}

A TCI tem sido desenvolvida ao longo das últimas três décadas como uma ferramenta para abordagem de problemas psicossociais. Nesse período desenvolveu-se também um corpo de pesquisas acadêmicas voltados para o tema, o que é estimulado pelo próprio criador da $\mathrm{TCI}$ no manual que serve para a formação e apoio na prática dos terapeutas comunitários. Neste consta um capítulo com instrumentos pré-formatados para a coleta de dados a fim de verificar os vínculos sociais, autoestima e impacto coletivo da $\mathrm{TCl}$, além de fichas para apreciação da atuação na roda pelos terapeutas que a conduziram e para organização das informações sobre a própria realização da roda (local, participantes, tema e estratégias de enfrentamento), sugeridos como uma forma de avaliar o impacto da TCl. Assim, Barreto enfatizou a necessidade de avaliar a TCI tanto no sentido da reflexão dos terapeutas sobre seu trabalho como a fim de qualificar suas bases teórica, metodológica e ética ${ }^{6}$.

Nesta revisão sistemática, foram identificados 168 diferentes registros sobre a temática da TCl. Porém, não foi possível identificar, dentre estes, nenhum estudo que avaliasse os efeitos da TCl em pessoas com TMC de forma longitudinal em comparação com quaisquer outras intervenções já estabelecidas. Inexistem, portanto, evidências que apontem para a efetividade da TCI na abordagem das pessoas com TMC na APS.

Dos 14 estudos consultados na íntegra que abordavam sujeitos com TMC em diferentes contextos do Sistema de Saúde e descreviam a TCl como uma forma de intervenção, identificaram-se dois com seguimento longitudinal, porém ambos foram excluídos. O primeiro deles não apresentava qualquer forma de controle, nem mesmo em relação aos próprios sujeitos antes da intervenção ${ }^{12}$. O segundo também não empregava grupo controle e, além disso, não apresentava avaliação dos resultados ${ }^{24}$. Entre os demais estudos analisados e excluídos, um deles foi realizado com grupo controle, porém sem seguimento longitudinal ${ }^{27}$. Neste, a conclusão de que idosos com depressão participavam menos da $\mathrm{TCl}$ do que aqueles sem depressão, na ausência do seguimento longitudinal, pode indicar tanto que os idosos participantes da $\mathrm{TCl}$ desenvolvam menos depressão como que aqueles idosos com depressão tenham menos disposição para participar das rodas de $\mathrm{TCl}$. 
A maioria dos estudos identificados empregou metodologias qualitativas, o que não invalidaria a avaliação quanto à efetividade da $\mathrm{TCl}$. O predomínio de pesquisas qualitativas parece simplesmente estar de acordo com a perspectiva epistemológica habitualmente atribuída aos profissionais e pesquisadores dedicados às PIC, dentre elas a TCl. No entanto, são facilmente identificadas na literatura revisões sistemáticas envolvendo vários estudos quantitativos com desenhos elaborados com a finalidade de avaliar a efetividade dessas práticas, em especial estudos randomizados controlados, que abordaram o uso de PIC em diferentes contextos, como a hipertensão arterial sistêmica, tratamento da dor ou seu uso em populações pediátricas ${ }^{30,31,32}$.

Porém, a absoluta maioria dos estudos aqui verificados foi desenvolvida de forma transversal, impedindo quaisquer comparações que pudessem indicar a efetividade da $\mathrm{TCI}$ na abordagem dos TMC. Esse aspecto limitante que surge na presente revisão pode refletir a falta de recursos de pesquisa dedicados às PIC, especialmente quando se trata de uma tecnologia leve-dura, que não desperta interesse por parte do complexo médicoindustrial. Estudos longitudinais, com critérios de inclusão e exclusão bem definidos, desfechos descritos claramente, seja de forma quantitativa ou qualitativa, constituiriam uma referência adequada para o emprego da TCI no SUS.

As bases legais do SUS definem que os produtos de interesse para a saúde deveriam ser avaliados quanto à sua eficácia, segurança, efetividade e custoefetividade. Uma vez incorporadas ao SUS, cabe ao Departamento de Ciência e Tecnologia (DECIT) da Secretaria de Ciência, Tecnologia e Insumos Estratégicos (SCTIE) apoiar o monitoramento de sua efetividade, sendo parte do sistema que acompanha o cumprimento dos objetivos do sistema, de acordo com as metas sanitárias e prioridades do SUS. Porém, habitualmente esse processo se dá de forma passiva, a partir de demandas da indústria farmacêutica, a qual conta com os recursos técnicos e financeiros para desenvolver os próprios estudos a fim de solicitar a inclusão ou a alteração de uma tecnologia. Desse modo, podem-se gerar inequidades que "[...] têm um impacto tanto na eficácia, pois o sistema não cumpre o objetivo de maximizar os benefícios à saúde, quanto na eficiência, ao promover o uso dos recursos [...] na avaliação de tecnologias potencialmente desvinculadas do perfil epidemiológico"32:29. A prioridade acaba sendo garantida para a avaliação e incorporação de tecnologias e insumos de interesse para o complexo médico-industrial, basicamente voltadas para a assistência com base em tecnologias duras, deixando em segundo plano intervenções voltadas para a prevenção de doenças ou promoção da saúde, como é o caso da $\mathrm{TCI}$.

Assim sendo, torna-se ainda mais relevante que os recursos limitados para pesquisas dedicados à $\mathrm{TCl}$ sejam direcionados de forma a potencializar tais esforços. Entendendo-se que o desenvolvimento da técnica passa por sua difusão, já estabelecida no Brasil e no exterior, com formação e atualização continuada dos terapeutas comunitários, o que acontece em eventos periódicos disponíveis, 
com a criação de sociedades e instituições dedicadas à $\mathrm{TCl}$, sua avaliação de forma estruturada e confiável seria um próximo passo já previsto na própria formação e qualificação dos terapeutas ${ }^{6}$.

Para isso, sugere-se que futuros estudos se ocupem da lacuna da longitudinalidade no desenho da metodologia, de forma que se utilizem comparações a fim de verificar se há efetividade na abordagem dos TMC com o emprego da TCl, sendo este um instrumento sugerido para os cuidados em saúde mental na APS. Ainda que haja limitações em termos de tempo e recursos de pesquisa para desenvolver um estudo com um grupo controle e randomização adequada, a análise de indicadores antes e após a intervenção com $\mathrm{TCl}$ no mesmo grupo de sujeitos ou o uso de controles em lista de espera para atendimento psicológico, por exemplo, podem ser empregados com a aplicação de escalas simples já padronizadas para sintomas de ansiedade ou depressão, constituindo um próximo passo essencial para que futuros estudos possam prover maior suporte teórico para a base empírica da TCl.

\section{REFERÊNCIAS}

1. Steel Z, Marnane C, Iranpour C, Chey T, Jackson JW, Patel V, Silove D. The global prevalence of common mental disorders: a systematic review and meta-analysis 1980-2013. Int J Epidemiol [Internet]. 2014 abr [citado em 2018 fev 25]; 43(2):47693. Disponível em: https://doi.org/10.1093/ije/dyu038.

2. National Collaborating Centre for Mental Health (UK). Common Mental Health Disorders: Identification and Pathways to Care [Internet]. 2011 [citado em 2018 mar 15]; Leicester (UK): British Psychological Society. Disponível em: https://www.ncbi. nlm.nih.gov/books/NBK92254/.

3. Ministério da Saúde (Brasil). Cadernos de Atenção Básica, n. 34 - Saúde mental. Brasília: Ministério da Saúde; 2013. 176 p.

4. Rüdell K, Bhui K, Priebe S. Do 'alternative' help-seeking strategies affect primary care service use? A survey of help-seeking for mental distress. BMC Public Health [Internet]. 2008 [citado em 2018 fev 25]; 8:207. Disponível em: http://www. biomedcentral.com/1471-2458/8/207.

5. Aires M, Roos CM, Gonçalves AVF, Schneider JF, Olschowsky A. Ações em saúde mental às famílias nos diferentes contextos de trabalho: revisão integrativa. Rev Gaúcha Enferm. 2010 set; 31(3):567-74.

6. Barreto AP. Terapia comunitária: passo a passo. 4. Ed. Rev e ampl. Fortaleza: Gráfica LCR; 2008.

7. Ministério da Saúde (Brasil). Portaria $n^{\circ} 849$ de 27 de março de 2017. Brasília, DF, 2017 mar. 
8. Lazarte L. Sociología y terapia comunitaria integrativa. Rev Urug Enferm. 2012 maio; $7(1): 67-76$.

9. Azevedo EB, Cordeiro RC, Costa LFP, Guerra CS; Ferreira Filha MO, Dias MD. Pesquisas brasileiras sobre terapia comunitária integrativa. Rev. Bras. Pesq. Saúde. 2013 julset; 15(3):114-20.

10. Ferreira Filha MO, Lazarte R, Barreto AP. Impacto e tendências do uso da Terapia Comunitária Integrativa na produção de cuidados em saúde mental. Rev. Eletr. Enf. [Internet]. 2015 abr-jun [citado em 2018 fev 25]; 17(2):172-3. Disponível em: http:// dx.doi.org/10.5216/ree.v17i2.37270.

11. Mourão LF, Oliveira LB, Marques ADB, Branco JGO, Guimarães MSO, Nery IS. Terapia comunitária como novo recurso da prática do cuidado: revisão integrativa. SANARE. 2016 jun-dez; 15(2):129-35.

12. Nascimento MVN, Leite CCA, Rodrigues BS, Santos IA, Oliveira JOD, Bitú LSL. Práticas Integrativas em Saúde: uma experiência da Terapia Comunitária na Atenção Básica. Rev enferm UFPE online [Internet]. 2012 out [citado em 2018 mar 15]; 6(10):2586-95. Disponível em: https://periodicos.ufpe.br/revistas/ revistaenfermagem/article/download/7506/7140.

13. Feitosa ANC, Garcia Filho C. Terapia Comunitária como Dispositivo de Cuidado em Saúde Mental na Atenção Básica. Anais. In: Seminário Internacional de Redes de Atenção à Saúde: Compartilhando Saberes e Práticas com interlocução na APS. 2016, Fortaleza [Internet]. 2016 [citado em 2018 fev 25]; Anais. Fortaleza: Universidade Estadual do Ceará; 2016. Disponível em: http://uece.br/eventos/ seminariosaudecoletiva/anais/trabalhos.html.

14. Cisneiros VGF, Oliveira MLS, Amaral GMC, Cunha DM, Silva MRF. Percepção dos Profissionais de Saúde e Comunitários em Relação à Terapia Comunitária na Estratégia Saúde da Família. Rev APS. 2012 out-dez; 15(4): 468-78.

15.Liberati A, Altman DG, Tetzlaff J, Mulrow C, Gøtzsche PC, et al. The PRISMA Statement for Reporting Systematic Reviews and Meta-Analyses of Studies That Evaluate Health Care Interventions: Explanation and Elaboration. PLoS Med [Internet]. 2009 [citado em 2017 out 10]; 6(7):e1000100. Disponível em: https://doi. org/10.1371/journal.pmed.1000100.

16. Soares DSCA. Terapia Comunitária na Estratégia Saúde da Família: implicações no modo de andar a vida dos usuários [dissertação]. Ribeirão Preto: USP; 2008.

17. Arruda AG. Saúde mental na comunidade: a terapia comunitária como dispositivo de cuidado [dissertação]. Fortaleza: UEC; 2010.

18. Cordeiro RC, Azevedo EB, Silva MSS, Ferreira Filha MO, Silva PMC, Moraes MN. Terapia Comunitária Integrativa na Estratégia Saúde da Família: análise acerca dos depoimentos dos seus participantes. Rev Univ Vale do Rio Verde [Internet]. 2011 [citado em 2018 mar 10]; 9(2):192-201. Disponível em: periodicos.unincor.br/index. php/revistaunincor/article/view/150. 
19. Fernandes MTO, Aquino AL, Santos GC, Soares SM, Lima LC. Terapia comunitária: Uma metodologia inovadora na Atenção Primária em Saúde no Brasil. Evidentia [Internet]. 2011 abr-jun [citado em 2018 mar 15]; 8(34). Disponível em: http://www. index-f.com/evidentia/n34/ev7494p.php.

20. Moura SG, Ferreira Filha MO, Cordeiro RC, Braga LAV, Monteiro CQA. A experiência da Terapia Comunitária em diferentes instituições e contextos populacionais. Revista UninCor [Internet]. 2012 [citado em 2018 mar 10]; 10(1): 329- 38. Disponível em: file://C:/Users/User/Downloads/Dialnet-AExperienciaDaTerapiaComunitariaEmDife rentesInstit-5033101.pdf.

21. Ferreira Filha MO, Sá ANP, Rocha IA, Silva VCL, Souto CMRM, Dias MD. Alcoolismo no contexto familiar: estratégias de enfrentamento das idosas usuárias da Terapia Comunitária. Rev Rede Enf Nord [Internet]. 2012 [citado em 2018 mar 15]; 13(1). Disponível em: http://www.redalyc.org/articulo.oa?id=324027980005.

22. Rocha IA, Ferreira Filha, MO. Contribuições da terapia comunitária integrativa sistêmica para a autonomia do idoso frente a situações adversas. In: IV CIEH, 2015, Campina Grande. Anais, Campina Grande: Centro Multidisciplinar de Estudos e Pesquisas (CEMEP), Universidade Estadual da Paraíba (UEPB) [Internet]. 2015 [citado em 2018 mar 10]. Disponível em: http://www.editorarealize.com.br/revistas/cieh/ trabalhos/TRABALHO_EV040_MD2_SA8_ID1632_27072015114501.pdf.

23. Nascimento ABM. Estigma, sociabilidade e práticas terapêuticas: a terapia comunitária como âncora e alívio do transtorno mental [dissertação]. Recife: Universidade Federal de Pernambuco; 2015.

24. Silva PMC. A TC como estratégia de intervenção para empoderamento dos usuários do CAPS em processo de alta [tese]. João Pessoa: UFPB; 2016.

25. Feitosa ANC, Garcia Filho C. Terapia comunitária como dispositivo de cuidado em saúde mental na atenção básica. In: Seminário internacional de redes de atenção à saúde, 2016, Fortaleza. Anais, Fortaleza: Programa de Pós-Graduação em Saúde Coletiva (PPSAC), da Universidade Estadual do Ceará (Uece) [Internet]. 2016 [citado em 2018 mar 10]. Disponível em: http://uece.br/eventos/seminariosaudecoletiva/ anais/trabalhos.html.

26. Lima Silva V, de Medeiros CAC, Guerra GCB, et al. Psychiatr Q [Internet]. 2017 [citado em 2018 mar 15]; 88:359. Disponível em: https://doi.org/10.1007/s11126016-9453-z.

27. Lutterbach MGC. A contribuição da terapia comunitária integrativa (TCI) na produção do cuidado e formação em saúde na atenção psicossocial [dissertação]. Niterói: Universidade Federal Fluminense; 2017.

28. Silva BCC, Lutterbach MGC, Arão S, Pinheiro Z. A experiência de rodas de Terapia Comunitária Integrativa (TCl) em CAPS (Centros de Atenção Psicossocial) e em ESF (Estratégia de Saúde da Família) nos municípios de Cantagalo e Nova Friburgo (RJ). Temas em Educação e Saúde [Internet]. 2017 [citado em 2018 maio 27]; 9:25263471. Disponível em: https://periodicos.fclar.unesp.br/tes/article/view/9585. 
29. Nahas R. Complementary and alternative medicine approaches to blood pressure reduction: An evidence-based review [Internet]. 2008 nov [citado em 2018 maio 27]; 54(11):1529-33. Disponível em: https://www.ncbi.nlm.nih.gov/pubmed/19005120.

30. Hunt K, Erns E. The evidence-base for complementary medicine in children: a critical overview of systematic reviews. Arch Dis Child [Internet]. 2011 [citado em 2018 maio 27]; 96:769-76. Disponível em: https://www.ncbi.nlm.nih.gov/ pubmed/20605859.

31. Dhanani NM, Caruso TJ, Carinci AJ. Complementary and Alternative Medicine for Pain: An Evidence-based Review. Curr Pain Headache Rep [Internet]. 2011 [citado em 2018 maio 27]; 15:39-46. Disponível em: https://www.ncbi.nlm.nih.gov/ pubmed/21063917.

32. Araújo DV, Distrutti M, Elias F. Priorização de tecnologias em saúde: o caso brasileiro. J. bras. econ. saúde (Impr.). 2017 set; 9(Suppl. 1):4-40.

Submissão: julho de 2018.

Aprovação: julho de 2020. 